\title{
Development and Control of a Differential Wall Climbing Robot based on Vortex Adhesion
}

\author{
Andreas Papadimitriou, George Andrikopoulos, George Nikolakopoulos
}

\begin{abstract}
In this article, the development and control of a novel differential drive Vortex Robot (VR) will be presented. Towards the direction of developing a climbing robot for inspection and repair of large infrastructures, a lightweight reliable climbing robot is proposed, being able to carry high payload via an Electric Ducted Fan (EDF) vortex based adhesion actuator. Towards these objectives, the fundamental elements of the overall design will be presented. For the preliminary evaluation of the proposed system, a PID-based control architecture will be analyzed and experimentally evaluated, with the goal of controlling the locomotion properties of the VR, while achieving a constant adhesion regardless the orientation of the robot and the surface's inclination. To further evaluate the robot's efficiency in real-life scenarios, such as the inspection and repair of airplane structures, results acquired via field trials involving a full-scale Boeing 737 will be presented.
\end{abstract}

\section{INTRODUCTION}

Recently, the area of wall climbing robots has received a lot of research attention mainly for inspection purposes, while their locomotion and operating properties are enabling novel applications in the maintenance sector [1], [2]. These robots base their fundamental operation in different methods for adhesion, while the main categories can be summarized as: a) passive [3], [4] and active suction cups [5], [6], b) vortex chambers [7], [8], and c) magnetic attraction [9], [10].

From all the different adhesion methods, vortex generation enabled via Electric Ducted Fan (EDF)-based designs, has the ability to provide important advantages. Specifically, designs incorporating EDFs as adhesion actuators enhance the climbing ability on various surface types, where other solutions have material-based limitations e.g. magnetic-based adhesion [11] with the inherent drawback of not supporting operation in non-ferromagnetic surfaces. In addition, EDFbased adhesion enables non-contact surface attachments, thus making it suitable for Non Destructive Testing (NDT) while avoiding the possibility of causing damages due to high contact pressure as, for example, applied in cases of suction cup-enabled adhesion. In contrast to robots that use suction cups like [12], EDF-based solutions have in principle a controllable adhesion level, thus enabling a continuous motion without constraining the speed or the mechanical need for attachment/detachment.

However, despite the challenge of deploying a control scheme for optimizing the generally high power consumption

This work has received funding from the European Unions H2020 Framework Programme under the call FET-OPEN, Grant Agreement No. 665238 .

The authors are with the Robotics Team at the Department of Computer Science, Electrical and Space Engineering at Luleå University of Technology, Luleå, Sweden

Corresponding Author's email: andpapeltu.se levels of EDFs, such an actuation method provides a high adhesion to weight ratio recently documented at approx. 9 [13] [14], thus providing a sufficient payload for carrying heavy equipment. The aforementioned advantages have an overall strong impact on wall climbing robots designs that could increase their potential usability in multiple application scenarios. In previous work, as presented in [14] and [15], the concept of vortex adhesion was investigated from an actuation point and extended by considering the thrust vectoring capabilities of a tilting vortex actuator.

The novelty and contribution of this article is triple. First, it analytically presents and analyzes a revised Vortex Robot (VR) design with a differential drive locomotion and the ability to dynamically control the force needed for a continuous adhesion, regardless of the surface and contact properties, i.e. curvature, inclination, friction etc.. The second contribution stems from the effort to increase the permissible payload, as an inherent principle from the proposed design, thus allowing the mount of inspection or/and repair tools. In this way, by providing a force sensing design, the adhesion level can be controlled dynamically based on the application needs, while the proposed locomotion method provides a versatile solution on path planning and trajectory design with an overall focus on the challenging surfaces from an inclination point. The final third contribution is the experimental evaluation sequences of the proposed design, through extensive laboratory trials involving flat surfaces of different inclinations, as well as its preliminary evaluation through field trials on different sections of a full-scale Boeing 737 airplane.

The rest of this article is structured as follows. In Section II, the overall design properties and components of the VR prototype are presented, while in Section III, the proposed cascaded PID-based control scheme is established. Section IV presents the results acquired from the laboratory and field experiments, while discussion points and concluding remarks are provided in Section V.

\section{VR PROTOTYPE DEVELOPMENT}

The VR prototype, depicted in Fig. 1, was designed with the primary goal of maximizing the payload that the robot can handle by utilizing a vortex actuator for achieving adhesion, while incorporating a differential-based design for enhancing the steering capabilities on the various inclination scenarios. The design of the VR focuses on minimizing the structural weight, without sacrificing the durability and reliability of the robot's components. Since the total adhesion forces generated from the utilized vortex actuator vary 


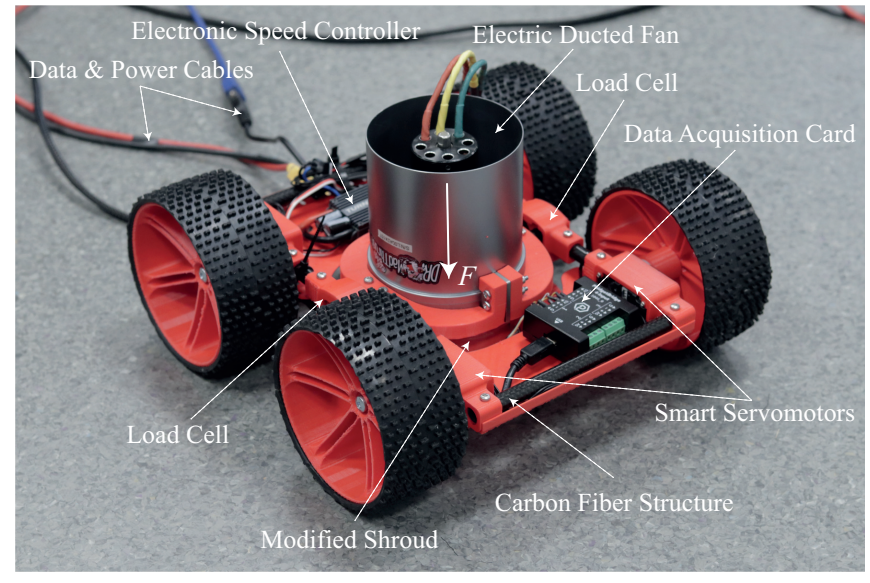

Fig. 1. The VR prototype with highlighted components.

from $0 \mathrm{~N}$ up to $80 \mathrm{~N}$ [14], to evaluate the ability of the robot to withstand such forces, iterative stress analyses were conducted to ensure that the structural quality of the involved components remains on the desired level.

Specifically and as shown in Fig. 1, the EDF is located in the middle of the robotic structure and it is supported from a ringlike frame, connected on the vehicle via carbon fiber tubes that interconnect in a casing for the load cells. The load cells are able to sense the level of the perpendicular to the surface exerted force of the EDF. Assuming that the generated force is distributed on the two load cells, which are the only points of contact between the EDF and the vehicle's frame, the total attractive force can be calculated from the summation of the individual load cell measurements. The EDF shroud is custom-designed and 3D printed for increasing the vortex generation, with dimensions chosen after an iterative sequence of design optimization for given robot dimensions and requirements posed from a set target application, as reported in [16]. These parts, interconnected via custom casings and carbon-fiber tubes, compose the VR's frame, while the wheels are connected to the servomotors via a modified horn.

The selected EDF on the presented study is a commercially available model with an inner duct diameter of $92 \mathrm{~mm}$, an overall weight $0.67 \mathrm{~kg}$ and a 12-blade fan, actuated via a brushless DC motor of $2350 \mathrm{~W}$ maximum power at 41000 maximum rpm, which provides a maximum free-flight thrust of $3.8 \mathrm{~kg}$, placed at the center of the robot. For enabling the utilization of such a robot in surfaces of variable curvatures, such as airplane fuselages, the methodology presented in [14] was utilized. Specifically, a shroud of $160 \mathrm{~mm}$ diameter profile was incorporated, which following the aforementioned methodology, leads to an attractive force of approximately $8 \mathrm{~kg}$. In the case of a flat surface, it has been documented in [14], that this maximum force is provided when the EDF is placed on the optimal distance from the projected surface, extracted at approximately $15 \mathrm{~mm}$. Larger forces can be achieved for the same EDF power, by following the aforementioned methodology regarding shroud size and distance from surface, but at a cost of robot specifications.
As far as the locomotion is concerned, four HerkuleX DRS-0101 smart servomotors were selected and mounted on a differential drive configuration, with the advantage of providing various feedback information, such as velocity, torque, position, temperature and respective overload limits, with a stall torque estimated at $12 \mathrm{~kg} . \mathrm{cm}$. The static friction constant between the utilized surface (paint-coated wooden boards) and the selected rubber was measured at $=0.82$.

The back and front of the VR structure was utilized for mounting electronic components, including an Electronic Speed Controller (ESC) for controlling the EDF, in this case the Turnigy AE-100A, and a Wheatstone Bridge Phidget DAQ 1500 for acquiring the force signals from the two FX1901 load cells placed on both sides of the vortex actuator (Fig. 1). The resulting dimensions of the robot are approximately $(272 \times 288 \times 150 \mathrm{~mm})(L \times W \times H)$ and with an overall weight of $1.983 \mathrm{~kg}$.

Finally, an external power supply was utilized for powering the VR, the acquisition of the setups sensorial data and the control of the EDFs operation was achieved via one National Instruments USB-6008 card and one Arduino Mega board, while the main control and programming of the setup was performed in a ground workstation (Windows 7, 64-b, Intel i7 processor, 32-Gb RAM).

\section{CONTROL SChEME FOR AdHesion AND LOCOMOTION}

The Degrees Of Freedom (DOFs) for the VR are presented in Fig. 2, where four active rotational joints $R_{i}, i \in\{1, \ldots, 4\}$ are manipulated from the smart servos, with controllable angular velocities $\omega_{i} \in \mathfrak{R}^{4}$ and are able to steer the vehicle properly with a velocity $v \in \Re$ and a heading angle $\beta \in \Re$. The EDF is locked in place in the center of the design, with the exerted force $F \in \mathfrak{R}$ being perpendicular to the projected surface. The Center of Gravity $(\mathrm{CoG})$ is assumed to be static and one of the design goals was to bring it as close to the wheel rotational plane as possible, while its distance from the surface is governed by the vortex actuator as the heaviest

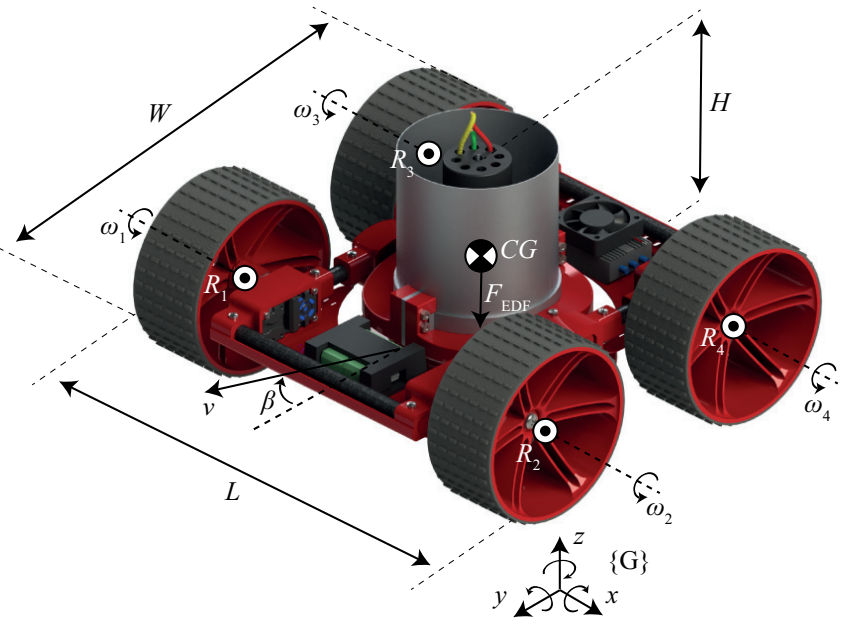

Fig. 2. Overview of the VR Degrees-of-Freedom (DOFs) and main geometrical components with respect to the reference frame $G$. 
component of the setup.

\section{A. Kinematics}

As previously mentioned, the VR is a differential skidsteering robot with four motorized wheels and with corresponding rotation speeds denoted as: $\omega_{1}=\omega_{3}=\omega_{R}$ and $\omega_{2}=\omega_{4}=\omega_{L}$, where $\omega_{R}$ and $\omega_{L}$ are the angular velocities of the right and left wheels respectively. Under the assumption of a rotational motion without wheel slippage, the relation that connects the wheel right and left linear velocities $v_{R}$ and $v_{L}$ to the angular velocities can be expressed as:

$$
\left[\begin{array}{l}
v_{R} \\
v_{L}
\end{array}\right]=\left[\begin{array}{l}
\omega_{R} \\
\omega_{L}
\end{array}\right] R
$$

where $R$ is the wheel radius. The total linear and angular velocity of the vehicle, with respect to the $\mathrm{CoG}$, can be calculated as shown in 2 , where $L$ is the distance between the left and right wheels:

$$
\left[\begin{array}{c}
v \\
\omega
\end{array}\right]=\left[\begin{array}{l}
\frac{v_{R}+v_{L}}{2} \\
\frac{v_{R}-v_{L}}{L}
\end{array}\right]
$$

The state vector for the VR is the generalized $2 D$ pose vector, as depicted in Fig. 2 and denoted as: $\mathbf{q}:=[x, y, \beta]^{T} \in \mathfrak{R}^{3}$, where the coordinates $x, y$ indicate the position of the robot on the $2 D$ plane and $\beta$ is the corresponding orientation. The vehicle's coordinates' rate of change can be expressed as:

$$
\left[\begin{array}{c}
\dot{x} \\
\dot{y} \\
\dot{\beta}
\end{array}\right]=\left[\begin{array}{cc}
\cos \beta & 0 \\
\sin \beta & 0 \\
0 & 1
\end{array}\right]\left[\begin{array}{c}
v \\
\omega
\end{array}\right]
$$

while the kinematic model can be expressed from the combination of (1), (2) and (3) as:

$$
\left[\begin{array}{c}
\dot{x} \\
\dot{y} \\
\dot{\beta}
\end{array}\right]=\left[\begin{array}{ll}
R & R \\
\frac{2}{2} \cos \beta & \frac{2}{2} \cos \beta \\
R & R \\
\frac{R}{2} \sin \beta & \frac{-}{2} \sin \beta \\
R & R \\
\frac{R}{L} & -\frac{R}{L}
\end{array}\right]\left[\begin{array}{l}
\omega_{R} \\
\omega_{L}
\end{array}\right]
$$

\section{B. Locomotion control}

For evaluating the motion and steering capabilities, a P-PIbased control scheme was utilized with a PI control for the velocity and a $\mathrm{P}$ control for the steering (Fig. 3). The purpose of these controllers is to adjust the control signal of the four wheels and lead to successful steering for enabling the VR's tracking of any set trajectory way-points. Specifically, given a reference point $p_{\text {ref }}=\left(x_{\text {ref }}, y_{\text {ref }}\right)$ in $\{O\}$ and the VR's current coordinates $p=(x, y)$ in $\{O\}$, the heading angle reference $\beta_{\text {ref }} \in \mathfrak{R}$ is computed as shown in (5). The heading error $e_{\beta} \in\left[e_{\beta}^{-}, e_{\beta}^{+}\right]=[-\pi, \pi]$ where $(.)^{-},(.)^{+}$denote lower bound and upper bound respectively is given in (6). To regulate the left and right wheels' angular velocities for achieving the desired steering, a $\mathrm{P}$ controller is tuned in the form of (7), where $K_{p, 1} \in \mathfrak{R}^{+}$is the controller's proportional gain.

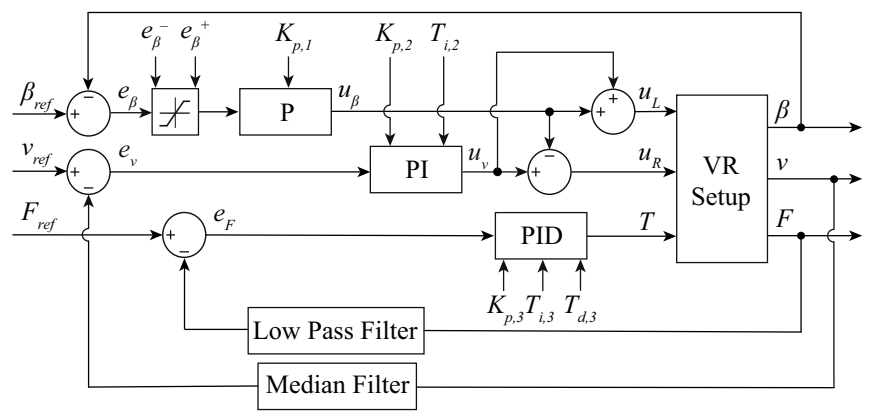

Fig. 3. Overall block diagram of the system.

$$
\begin{aligned}
\beta_{r e f} & =\operatorname{atan} 2\left(\left(y_{r e f}-y\right),\left(x_{r e f}-x\right)\right) \\
e_{\beta} & =\beta_{r e f}-\beta \\
u_{s}(k) & =K_{p, 1} e_{\beta}(k)
\end{aligned}
$$

where the velocity profile is determined offline and is provided to the system as a reference value $v_{r e f} \in \mathfrak{R}$. The velocity error is calculated as the difference of the desired velocity and the current velocity of the $\operatorname{VR} e_{v}=v_{\text {ref }}-v$, where $v \in \mathfrak{R}$ is the feedback velocity and calculated from (8) based on VR's position in space.

$$
v(k)=\frac{\sqrt{d_{x}(k)^{2}+d_{y}(k)^{2}}}{T_{s}}
$$

where $d_{i}(k)=[i(k)-i(k-1)]$ for $i=x, y$ shows the distance traveled on $x, y$ and $T_{s}$ is the sampling time. The PI controller, as depicted in Fig. 3, generates the control signal $u_{v}$ in 9 for regulating the velocity of the left and right wheels, where $K_{p, 2}, T_{i, 2} \in \mathfrak{R}^{+}$are the PI's tuning parameters.

$$
u_{v}(k)=K_{p, 2}\left[e_{v}(k)+\frac{1}{T_{i, 2}} \sum_{n=0}^{k} e_{v}(k)\right]
$$

The output signals from the P and PI controllers or $u_{\beta}$ and $u_{v}$ respectively, form the right and the left wheels inputs as $u_{R}=$ $u_{v}-u_{\beta}$ and $u_{L}=u_{v}+u_{\beta}$. The $u_{L}, u_{R} \in \mathfrak{R}$ are the input signals for the left and right wheels' motors, while the input to the motors is unit-less and the internal PID of the smart servos regulates the angular velocity of the wheels. To evaluate the control algorithm of the locomotion, different paths will be generated offline to evaluate the overall VR's performance. In this case, the path is point by point generated as a reference position, while this update at the $k$ time instant depends on the distance $d(k)$, measured from 10, of the current position $P(k)$ from the $P^{d}(k+1)$ and has to satisfy a tuning parameter $d_{\circ}$ i.e. if $d_{\circ} \leq d$ then $x_{r e f}(k)=x_{r e f}(k+1)$ and $y_{r e f}(k)=$ $y_{\text {ref }}(k+1)$, where $P^{d}(k+1)$ denotes the next desired point.

$$
d=\sqrt{\left(x_{r e f}-x\right)^{2}+\left(y_{r e f}-y\right)^{2}}
$$

\section{Adhesion control}

For controlling the adhesion force, the corresponding control scheme is presented in Fig. 3. In this case, the minimum force needed for successful adhesion, is the one that will 
allow the VR to remain static or move without slipping or falling and it depends on several factors, such as the total weight of the robot, surface type, cable tension etc..

Thus, the reference force $F_{r e f} \in \mathfrak{R}$ are extracted via simulation trials and provided as a look-up table in the adhesion controller of the VR as it will be analyzed in Section IV. The VR's PID error signal is calculated as the difference of the desired force and the measured force or $e_{F}=F_{r e f}-F$, where $F \in \mathfrak{R}$ is the filtered measurement from the load cells. The control action of the discrete adhesion PID controller is given in (11), where $K_{p, 3}, T_{i, 3}, T_{d, 3} \in \mathfrak{R}^{+}$are the PID's terms.

$$
T(k)=K_{p, 3}\left[e_{F}(k)+\frac{1}{T_{i, 3}} \sum_{n=0}^{k} e_{F}(k)+T_{d, 3} \frac{e_{F}(k)-e_{F}(k-1)}{d t}\right]
$$

The control signal $T \in \mathfrak{R}$ is the throttle level defined as a percentage from $0 \%$ to $100 \%$ and it is provided to the ESC controller for regulating the EDF's operation.

\section{EXPERIMENTAL EVALUATION}

\section{A. Lab experiments}

For the preliminary evaluation of the VR, experimental trials were conducted under different surface inclinations (Fig. 4). The purpose of these experiments was to exploit the adhesion properties for increasing the operation bandwidth and permissible payload, which will allow future incorporation of sensors and tools for conducting inspection and repair tasks. These experiments included closed-loop tests, with the robot placed on a surface inclined by (a) $90^{\circ}$ and (b) $180^{\circ}$, as shown in Fig. 4, tracking a predefined motion pattern in each scenario.

The required adhesion force $F_{\text {ref }}$ was set at $45 \mathrm{~N}$ and $40 \mathrm{~N}$ for the aforementioned (a) and (b) inclination cases respectively. These values were extracted from the dynamic simulation studies performed in Simscape Multibody. Additionally, the PID coefficients controlling the adhesion force were fine-tuned under iterative tests. In addition, the velocity and steering control of the vehicle were tuned to track the desired trajectories. For both selected inclination scenarios, an S-shaped path was utilized and the fine-tuned values of the PI and P controller for the velocity and steering.

It has to be noted that due to vibrations inserted from the EDF during operation, a $2^{\text {nd }}$ order low-pass filter was applied on the force feedback with cut-off frequency of $8 \mathrm{~Hz}$, while the velocity feedback was filtered with a windowed $3^{\text {rd }}$ rank median filter. In addition, the coefficient $d$ for updating the path points was tuned and kept constant at $d=0.1 \mathrm{~m}$, while the localization of the robot in the global frame was performed via the VICON motion capture system.

For both investigated cases, as presented in Fig. 5 (a) and (b), the VR is characterized by smooth motion and maneuverability, with successful tracking of the reference signals. In all presented time responses, it can be observed that the motion performance begins at approximately $2 \mathrm{sec}$, a delay posed for ensuring stable adhesion. As far as the adhesion performance is concerned, the PID controller was able to remain robust against the amplification noise in the

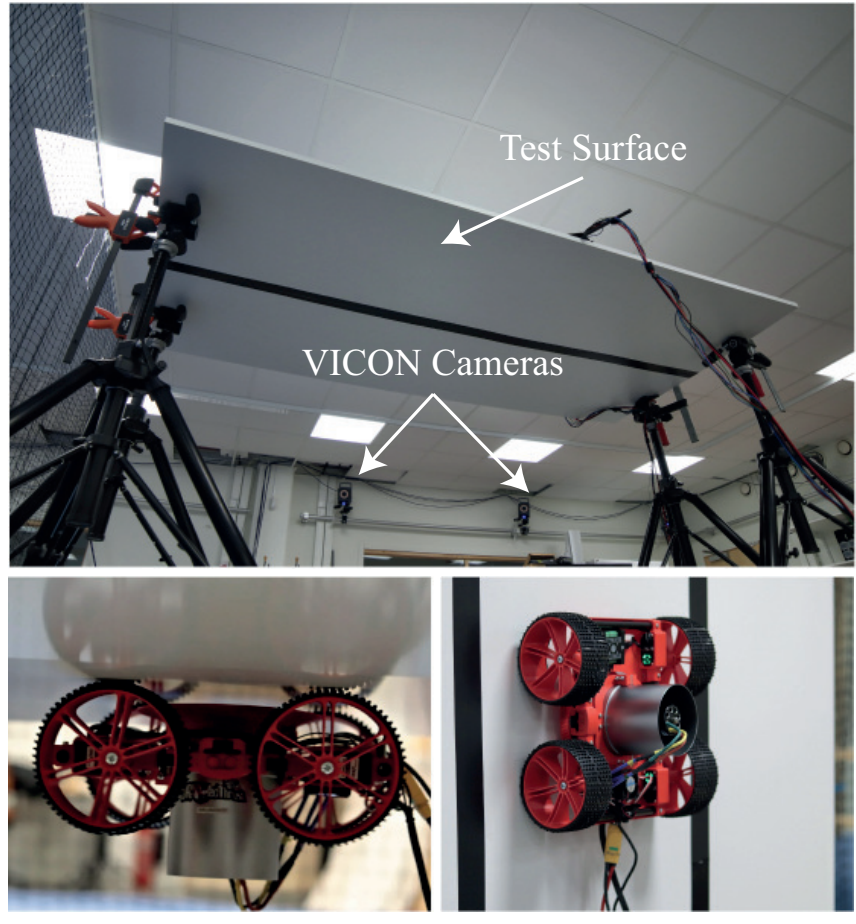

Fig. 4. Photographic stills of the test surface setup (top) utilized for the experimental evaluation of the VR, under inverted (bottom left) and vertical (bottom right) surface inclination scenarios

force feedback and keep the force close to the reference value. In the same 5 (a) and (b), it can be noticed that during both inclination scenarios the motion of the vehicle does not affect the adhesion force, due to the constant optimal distance of the EDF from the surface.

Regarding the VR's tracking performance, in case (a), tracking deviations could be observed at specific segments of the reference path, where the path changes required higher maneuverability. In addition, deviations can also been observed in the beginning of the acquired position responses until the robot is capable of overcoming the static friction and initialize its motion. During case (b), the VR path tracking response shows similar results in the beginning, with decreased tracking error for the rest of the task.

From the obtained responses, it can be also identified that the heading angle error, as depicted in Fig. 5 (a) and (b), converges towards zero and in a short time window. The error has the maximum values when the VR is at the most challenging parts of the path, which are the ones with the highest curvature changes. Also, it can be observed that the heading angle error has higher values on the vertical experiment since in this case the slippage effect is greater.

The velocity reference speed for the vertical experiments varies depending on the way-points of the path from 0 to $0.2 \mathrm{~m} / \mathrm{s}$, while for the inverted experiments the velocity kept at the constant rate of $0.1 \mathrm{~m} / \mathrm{s}$. As illustrated in Fig. 5 (a) and (b), the VR's velocity controller successfully manages to keep the velocity on the desired levels with small fluctuations on the steady state, mainly due to the existence of noise and small fluctuations during stiff turns of the selected path. 

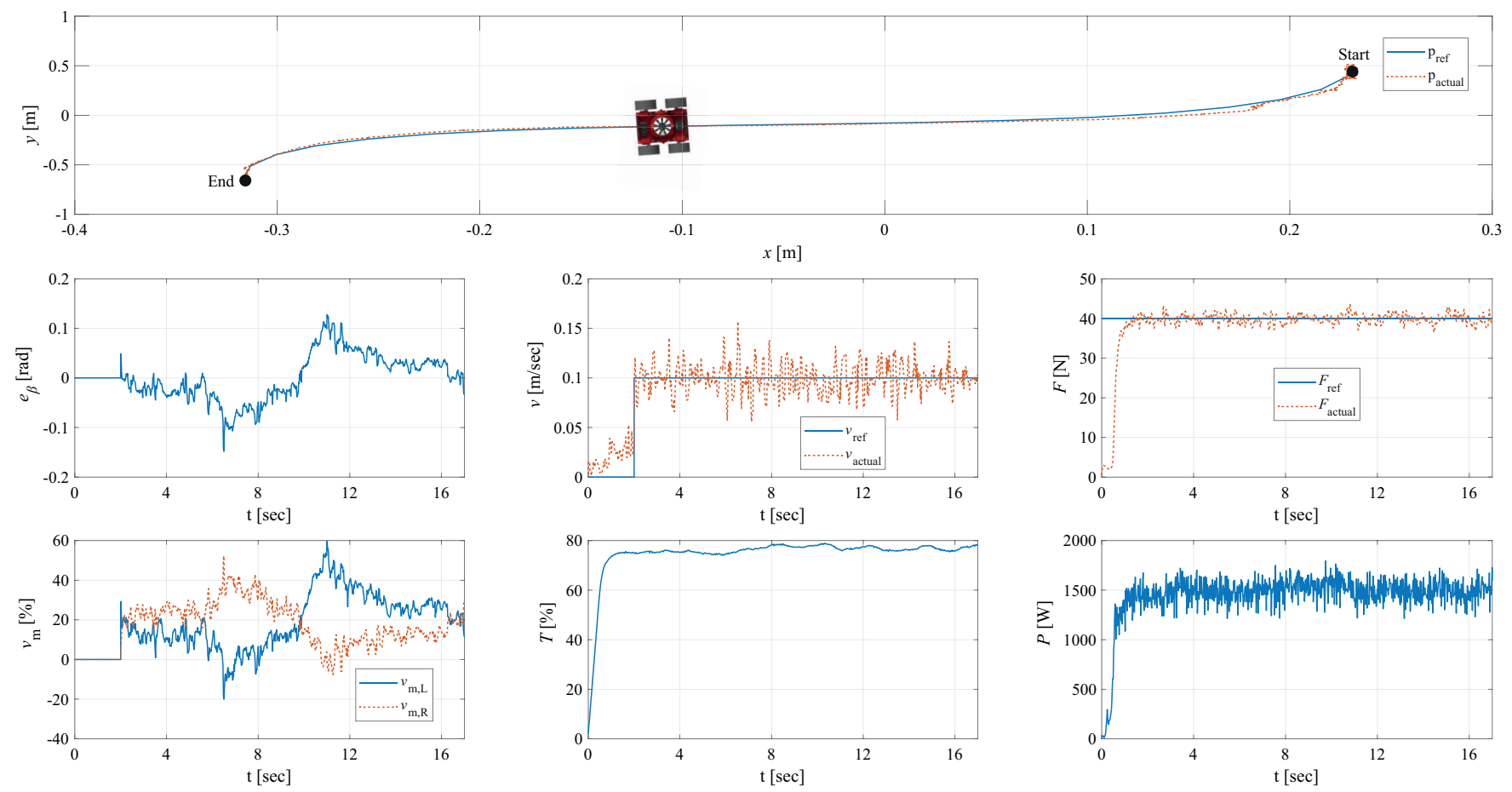

(a)
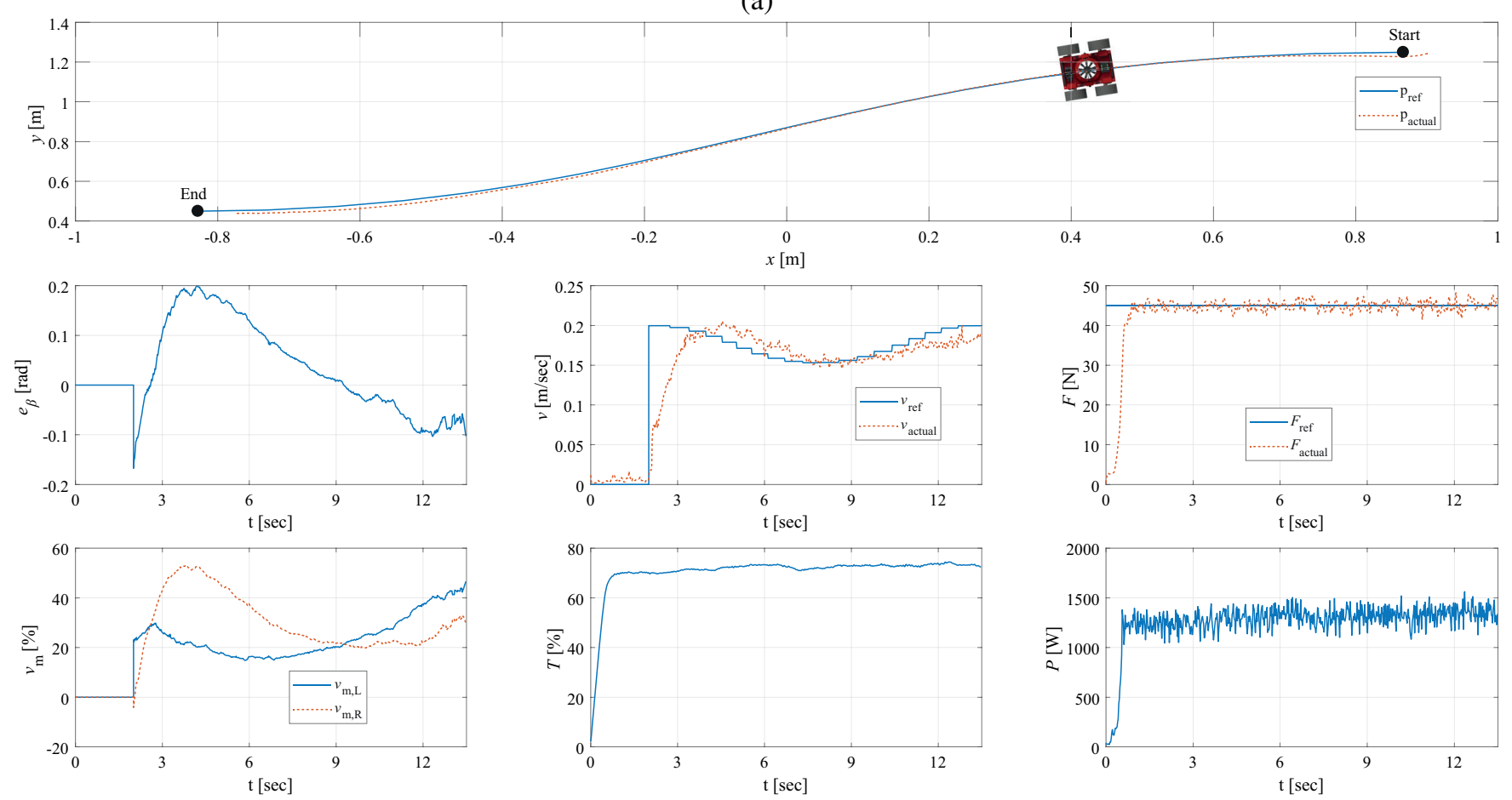

(b)

Fig. 5. VR's path following response for the inverted (a) and vertical (b) case, along with the respective time responses of the velocity $v$, heading angle error $e_{\beta}$, force $F$, power consumption $E_{a d}$, throttle $T$ and motors' input $v_{m}$.

Finally, Fig. 5 (a) and (b), also includes the measured power consumption $E_{a d}$. Since the experiments were performed at predetermined surface inclinations, the reference forces were kept at selected constant values for ensuring adhesion without utilizing the maximum power provided by the EDF. For that reason, it can be noticed that the power consumption remains near the level of $1200 \mathrm{~W}$ for the experiments on the vertical surface, while for the inverted experiments the power consumption was close to $1450 \mathrm{~W}$.

\section{B. Field experiments}

In an effort to evaluate the VR's performance in real-life test cases, a series of field-trials were performed where the 
VR was operated on different parts of a Boeing 737. Specifically, multiple open-loop test operations were executed at Cranfield University, United Kingdom, for testing the VR's ability to remain adhered on top of the various Boeing surfaces, regardless of orientation and inclination and while performing manually controlled patterns. As it is depicted in Fig. 6, the utilized tethered setup was controlled via a base station, including a workstation (laptop), microprocessors for acquiring and transferring sensor and actuator signals between the VR and the workstation.

\section{CONCLUSION}

This article presented a novel Vortex Robot (VR) from a design, development and experiment evaluation perspective. The study examined the potential use of an Electric Ducted Fan (EDF) as the main actuation unit for adhesion with the goal of maximizing the permissible payload the robot can handle. For the preliminary experimental evaluation of the VR prototype, a PID controller was selected for the control of adhesion, while for the transnational motion and the steering, a PI-P based structure was implemented. The executed experimental trials showed that the bandwidth of the permissible payload remained in the range of $3.1 \mathrm{~kg}$ to $5.9 \mathrm{~kg}$, while providing successful tracking of set paths via fast and accurate motion responses with smooth alterations even in cases of high required maneuverability. To further extend the evaluation on real-life scenarios, the VR was deployed on a full scale Boeing 737, where it successfully managed to maintain its adhesion and motion capabilities on the alternate curvatures of the airplane, while managing the challenging transition between the airplane wings and fuselage.

\section{ACKNOWLEDGMENTS}

The authors would like to thank Prof. Iain Gray, Mr. Joao Raposo, Dr. Luca Zanotti Fragonara, Dr. Janardhan Padiyar, Dr. Ivan Petrunin and Mr. Vasilis Tzitzilonis of Cranfield University for their help during the VR field trials.

\section{REFERENCES}

[1] Y. K. Song, C. M. Lee, I. M. Koo, D. T. Tran, H. Moon, and H. R. Choi, "Development of wall climbing robotic system for inspection purpose," in 2008 IEEE/RSJ International Conference on Intelligent Robots and Systems, IROS, 2008.

[2] L. Yangi, G. Yang, Z. Liu, Y. Chang, B. Jiang, Y. Awad, and J. Xiao, "Wall-climbing robot for visual and GPR inspection," Proceedings of the 13th IEEE Conference on Industrial Electronics and Applications, ICIEA 2018, pp. 1004-1009, 2018.

[3] C. Hillenbrand, D. Schmidt, and K. Berns, "CROMSCI: development of a climbing robot with negative pressure adhesion for inspections," Ind. Robot An Int. J., vol. 35, no. 3, pp. 228-237, 2008.

[4] Y. Guan, H. Zhu, W. Wu, X. Zhou, L. Jiang, C. Cai, L. Zhang, and H. Zhang, "A Modular Biped Wall-Climbing Robot With High Mobility and Manipulating Function," IEEE/ASME Trans. Mechatronics, vol. 18, no. 6, pp. 1787-1798, 2013.

[5] Z. Jiang, "Study on pneumatic wall climbing robot adhesion principle and suction control," 2008 IEEE Int. Conf. Robot. Biomimetics, pp. 1812-1817, 2009.

[6] J. Shang, T. Sattar, S. Chen, and B. Bridge, "Design of a climbing robot for inspecting aircraft wings and fuselage," Ind. Robot An Int. J., vol. 34, no. 6, pp. 495-502, 2007.

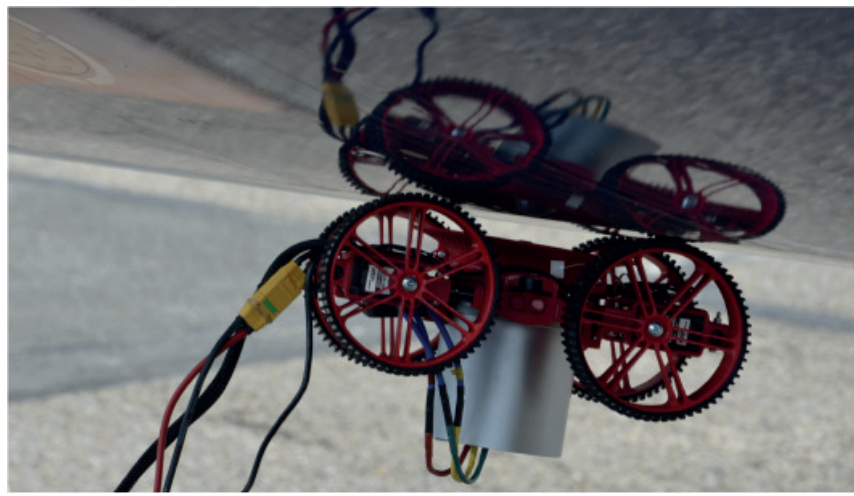

(a)

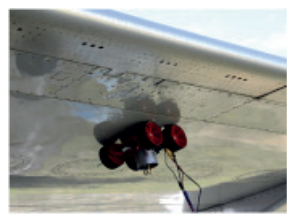

(b)

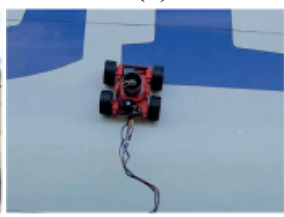

(c)

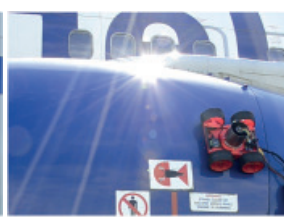

(d)

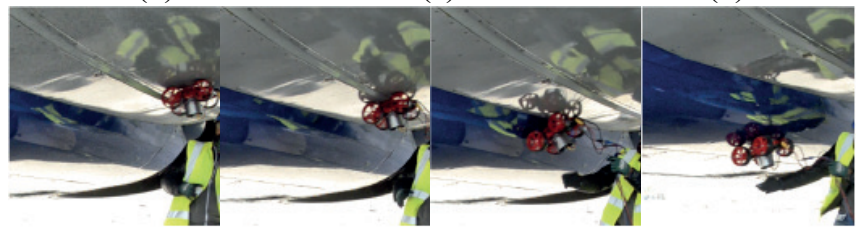

(e)

Fig. 6. Photographic stills of the VR prototype while attached and moving under varying orientations on the surfaces of the Cranfield Boeing 737: (a-b) fuselage, (c) wing, (d) turbine engine (e) fuselage to wing transition.

[7] B. Chu, K. Jung, C. S. Han, and D. Hong, "A survey of climbing robots: Locomotion and adhesion," Int. J. Precis. Eng. Manuf., vol. 11, no. 4, pp. 633-647, 2010.

[8] H. R. Choi, S. M. Ryew, T. H. Kang, J. H. Lee, and H. M. Kim, "A wall climbing robot with closed link mechanism," Proceedings. 2000 IEEE/RSJ Int. Conf. Intell. Robot. Syst. (IROS 2000), vol. 18, no. 4 pp. 2006-2011, 2000.

[9] M. Minor, H. Dulimarta, G. Danghi, R. Mukherjee, R. Lal Tummala, and D. Aslam, "Design, implementation, and evaluation of an under-actuated miniature biped climbing robot," Proceedings. 2000 IEEE/RSJ Int. Conf. Intell. Robot. Syst. (IROS 2000), vol. 3, pp. 1999 2005, 2000

[10] S. Hirose, A. Nagakubo, and R. Toyama, "Machine that can walk and climb on floors, walls and ceilings," Fifth Int. Conf. Adv. Robot. Robot. Unstructured Environ., vol. 1, pp. 753-758, 1991.

[11] M. Eich and T. Vögele, "Design and control of a lightweight magnetic climbing robot for vessel inspection," in 2011 19th Mediterranean Conference on Control and Automation, MED 2011, 2011.

[12] L. Technik, "MORFI," 2014. [Online]. Available: http://www.lufthansa-technik.com/en/tcd

[13] A. Brusell, G. Andrikopoulos, and G. Nikolakopoulos, "Novel considerations on the negative pressure adhesion of electric ducted fans: An experimental study," 2017 25th Mediterranean Conference on Control and Automation, MED 2017, pp. 1404-1409, 2017.

[14] G. Andrikopoulos and G. Nikolakopoulos, "Vortex Actuation via Electric Ducted Fans: an Experimental Study," Journal of Intelligent \& Robotic Systems, 92018.

[15] A. Papadimitriou, G. Andrikopoulos, and G. Nikolakopoulos, "Design , Development and Experimental Evaluation of a Thrust Vectoring Vortex Climbing Robot *," International Conference on Robotics and Biomimetics ROBIO, 2018.

[16] A. Brusell, G. Andrikopoulos, and G. Nikolakopoulos, "Novel considerations on the negative pressure adhesion of electric ducted fans: An experimental study," 2017 25th Mediterranean Conference on Control and Automation, MED 2017, pp. 1404-1409, 2017. 